\title{
Programmes lumineux conduisant à un étalement de la période de reproduction de l'oie
}

\author{
B. SAUVEUR \\ I.N.R.A., Station de Recherches avicoles, Centre de Recherches de Tours, \\ Nouzilly, F 37380 Monnaie
}

\begin{abstract}
Résumé
Dans les conditions d'éclairage naturel de la France, la ponte de l'oie intervient entre janvier et juin. Pour les deux sexes, l'âge à la maturité sexuelle est liée à l'évolution de la durée du jour à partir de la $20^{\circ}$ semaine. Chez le mâle la spermatogenèse est maximale entre février et avril ; la libido diminue à partir de cette date puis les animaux muent pendant l'été. La période photoréfractaire dure alors de 100 à 110 jours.

L'emploi de jours longs ou croissants permet d'avancer la maturité sexuelle de l'oie et du jars même s'ils n'interviennent qu'à partir de l'automne. A l'inverse, un éclairement décroissant puis croissant incluant un maintien intermédiaire en jours courts permet d'éviter une trop grande précocité sexuelle.

Les photopériodes de $14-15 \mathrm{~h}$ paraissent les mieux adaptées pour faire reprendre chaque année la ponte en fin d'automne alors que des valeurs plus élevées favorisent ensuite la couvaison. Des jours courts de $8 \mathrm{~h}$ utilisés en période de ponte permettraient au contraire de prolonger légèrement celle-ci et assureraient par ailleurs une meilleure persistance de production du sperme.

L'utilisation pendant l'été de jours courts de $7 \mathrm{~h}$ et d'une restriction alimentaire, suivie d'une stimulation lumineuse, permet d'obtenir une ponte d'automne-hiver comprise entre 10 à 25 cufs. Le temps de passage en jours courts semble devoir être voisin de 3 mois. Chez le mâle une telle exposition à des jours courts conduit à une bonne production de sperme assurant une fertilité élevée des oufs.

De façon plus générale, il est certainement possible d'obtenir, en bâtiment approprié, des successions de ponte séparées par des périodes de mue en jours courts mais il reste à évaluer les effets exacts des autres conditions climatiques (température notamment) sur la reproduction de l'oie en été.
\end{abstract}

\section{Introduction}

Durant la première moitié de notre siècle, le cheptel ansérin a régressé dans les pays où se développait l'élevage rationnel des autres volailles; c'est ainsi qu'aux Etats-Unis la production annuelle d'oisons a chuté de 8,5 millions d'unités en 1900 à 0,5 million en 1969. On assiste cependant depuis plusieurs années à une reprise de la production d'oisons à rôtir qui participent au phénomène de diversification des viandes de volailles offertes à la consommation. Cette production vient s'ajouter à celle d'oisons à gaver restée très active en France et dans l'Europe de l'Est. A 
l'heure actuelle on peut estimer aux environs de 2,5 millions d'unités la production annuelle contrôlée d'oisons en France, autour de 20 millions celle de l'ensemble Pologne, Hongrie, Tchécoslovaquie et à 20 millions également la production russe (KROPP, 1976).

Si l'on admet qu'une oie donne naissance à 30 oisons par an (chiffre très variable, en particulier selon les races), on peut estimer autour de 1,5 million de têtes le cheptel européen d'oies reproductrices.

Dans la plupart des cas ces oies ont encore accès à des parcours herbeux et/ou des bassins de copulation extérieurs et sont de ce fait soumises aux rythmes d'éclairements naturels; elles présentent donc une ponte de printemps, caractéristique de l'espèce. Un désaisonnement de cette production d'oisons est cependant souhaité de façon à mieux amortir les installations de gavage ou d'engraissement des jeunes. L'utilisation de programmes lumineux peut, dans cette optique, être envisagée de diverses façons :

\section{Chez la femelle} en ponte.

- Pour contrôler la date et les conditions techniques de la première entrée

- Pour étaler sur une plus longue période la ponte «classique» de printemps.

- Pour décaler au cours de l'année les démarrages de ponte de troupeaux d'âges différents présents sur une même exploitation.

- Pour obtenir qu'un même troupeau revienne en ponte après un repos estival.

- Dans une optique plus générale, pour obtenir des pontes successives totalement désaisonnalisées.

\section{Chez le mâle}

- Pour assurer une précocité sexuelle adaptée à celle des femelles.

- Pour maintenir une bonne persistance de la fertilité au cours des campagnes successives de reproduction; penser cependant que, sauf recours à l'insémination artificielle, les programmes lumineux sont en principe communs aux mâles et aux femelles.

Les lignes qui suivent sont un résumé des travaux trouvés dans la bibliographie sur ces différents points. Compte tenu de la diversité des conditions expérimentales rencontrées (race et âge des animaux, type de logement, critère retenu : poids de l'ovaire, nombre d'œufs, etc.) on veillera à ne pas extrapoler abusivement les résultats rapportés ici.

\section{Rappels sommaires sur la sensibilité de l'oie à l'évolution de la photopériode naturelle}

\subsection{Femelles}

Dans un élevage en lumière naturelle, la ponte de l'oie s'étend sous nos latitudes, pendant 15 à 22 semaines comprises entre le 15 janvier et le 15 juin environ (fig. 1 ). 


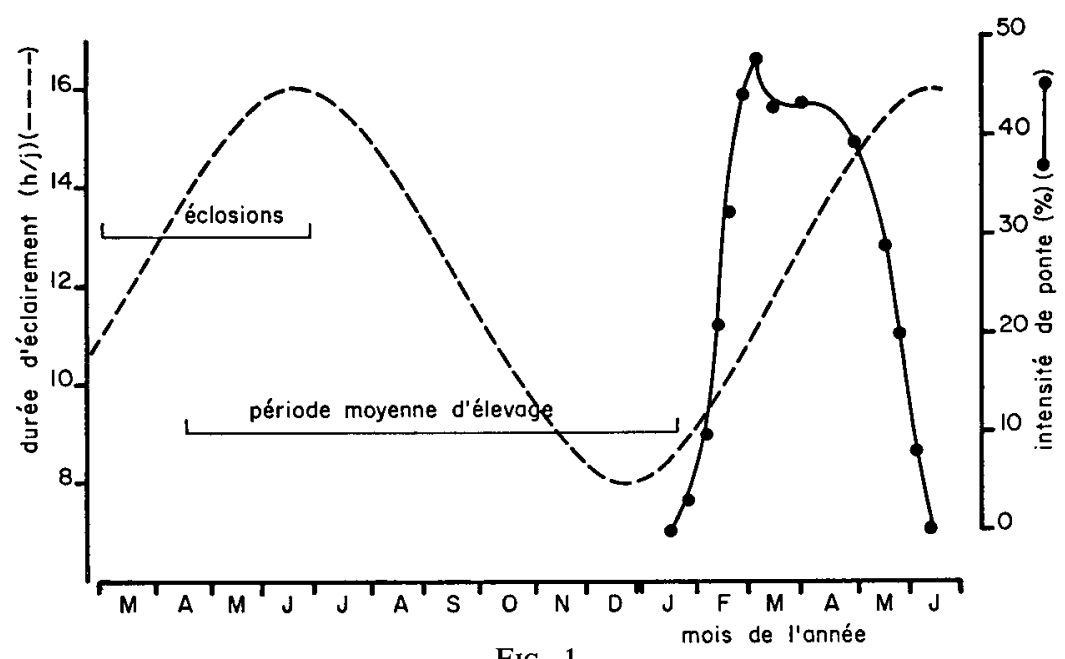

FIG. 1

Situation normale de la ponte de l'oie par rapport à l'évolution de la photopériode (oies landaises de 2-3 ans; données de la Station expérimentale d'Artiguères, 1971-1972).

Normal laying period of geese relative to photoperiod changes (2-3 year old «Landaise» geese $)$.

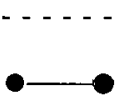

Durée du jour naturel $(\mathrm{h} / \mathrm{j})$.

Natural day length $(h / d)$.

Intensité de ponte.

Laying rate (p. 100).

La ponte de première année commence plus tard ( 2 à 3 semaines) et se termine plus tôt ( 2 semaines) que celles des années ultérieures (Merritr \& Lemay, 1963).

La maturité sexuelle se situe couramment entre la $30^{\circ}$ et la $50^{\circ}$ semaine d'âge selon la race et les conditions climatiques; elle est partiellement fonction de la date de naissance comme l'indique la figure 2 établie d'après les données de Borisov (1966) ; si l'on prend l'exemple des oies de Toulouse, il y apparaît que lorsque le mois de naissance change de février à juillet, l'âge au premier ouf diminue de 52 à 42 semaines et se place donc en mars et mai respectivement. Les animaux nés tardivement (octobre à janvier) sont extrêmement précoces et peuvent entrer en ponte en mai-juin entre 25 et 30 semaines d'âge.

L'examen de la figure 2 permet de se rendre compte que l'âge à la maturité sexuelle est peu influencé par l'évolution de la durée du jour durant les 4 ou 5 premiers mois qui suivent l'éclosion. Au contraire, l'oie, comme la poule, devient de plus en plus sensible à l'évolution de cette durée au fur et à mesure qu'elle vieillit ; sont donc précoces les animaux qui sont exposés à des jours croissants entre 20 et 40 semaines d'âge environ et tardifs ceux qui subissent des jours décroissants pendant la même période. 


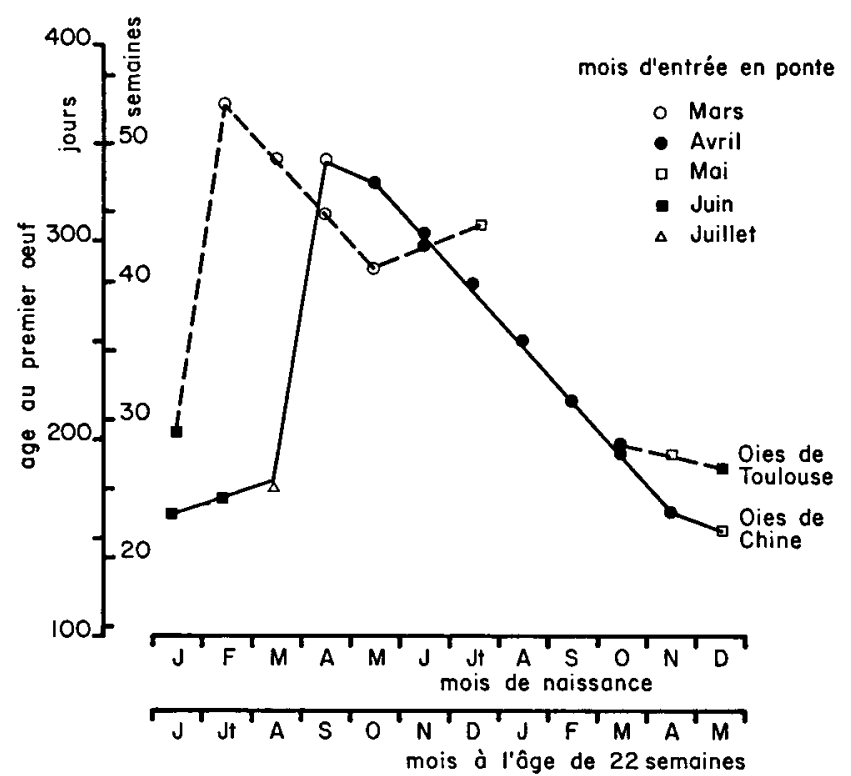

FIG. 2

Influence du mois de naissance sur la précocité sexuelle (âge au $1^{\text {er }}$ auf) chez l'oie (d'après BoRISOv, 1966).

Effect of birth month on the age at the first egg (days and weeks).

The line under abscissa indicates the month at 22 weeks of age in order to show the effect of increasing days at this stage.

\subsection{Mâles}

Chez le mâle né normalement au printemps et élevé en lumière naturelle, les premiers spermatocytes apparaissent à 18 semaines d'âge, les spermatides rondes à 24 semaines et les spermatozoïdes à 30 semaines (KLosowsKa, BielinsKA \& KLosowski, $1979 \mathrm{a}$ ). Le poids testiculaire atteint un plateau à 34 semaines d’âge chez des jars nés le 20 mai (Blagodatskaia, 1955).

L'activité sexuelle mâle estimée par le poids testiculaire, le diamètre des tubules et les rendements des différentes étapes de la spermatogenèse est maximale entre février et avril. En mai, on constate déjà des altérations de l'épithélium séminifère qui s'involue en juin (KlosowsKa, BielinsKa \& KlosowsKi, 1979 b).

Des mâles nés en novembre (cf. plus loin pour la justification de cette possibilité) et élevés en lumière naturelle atteignent leur maturité sexuelle à 24 semaines (début mai) au lieu de 30 (BoRYs, 1979). Les jours croissants ont donc un effet stimulant sur la précocité sexuelle du mâle comme celle de la femelle s'ils sont appliqués après 1 ou 2 mois de jours décroissants. On ignore quelle est alors la persistance de la fertilité à l'âge adulte et, en particulier, si ces mâles peuvent assurer une fertilisation satisfaisante des œufs durant l'été.

Après la période de reproduction, le jars, comme le canard, présente une période dite "réfractaire» pendant laquelle il est insensible aux stimulations lumineuses; selon Grom (1969), elle durerait de 14 à 15 semaines. 


\section{Réponses des oies femelles à différents programmes d'éclairement}

\subsection{De la naissance à l'entrée en ponte}

\subsection{Effets d'un éclairement constant}

Des jours constants de $9 \mathrm{~h}$ utilisés dès le jeune âge entraînent un important développement de l'ovaire entre 20 et 30 semaines suivi d'une stabilisation (BLAGoDATSKAIA, 1955); l'âge au premier œuf n'est pas inférieur à celui obtenu avec la lumière naturellement décroissante (BoRIsov, 1966; tabl. 1). A l'opposé, des jours de 12 ou $14 \mathrm{~h}$ sont fortement stimulants, et permettent d'avancer la ponte de 5 et 15 semaines respectivement chez l'oie de Toulouse (tabl. 1). Les auteurs ne précisent pas la production ultérieure des animaux.

\section{TABLEAU 1}

Effets de différents programmes lumineux d'élevage sur l'âge au premier auf chez l'oie (en semaines) (BoRIsov, 1966).

Effect of different lighting programmes on the age at the first egg (in weeks)

\begin{tabular}{|c|c|c|c|c|c|c|}
\hline \multirow[t]{2}{*}{$\begin{array}{l}\text { Race } \\
\text { Breed }\end{array}$} & \multirow[t]{2}{*}{$\begin{array}{c}\text { Témoin } \\
\text { lumière } \\
\text { du jour } \\
\text { Control } \\
\text { Natural } \\
\text { light }\end{array}$} & \multicolumn{3}{|c|}{$\begin{array}{l}\text { Programmes constants } \\
\text { dès la naissance } \\
\text { Constant lighting since the birth }\end{array}$} & \multicolumn{2}{|c|}{$\begin{array}{l}\text { Programmes } \\
\text { décroissants } \\
\text { jusqu'à } 13 \text { semaines } \\
\text { puis constants à : } \\
\text { Lighting programmes } \\
\text { decreasing between } 0 \\
\text { and } 13 \text { weeks of age } \\
\text { and then constant at : }\end{array}$} \\
\hline & & $9 \mathrm{~h}$ & $12 \mathrm{~h}$ & $14 \mathrm{~h}$ & $12 \mathrm{~h}$ & $14 \mathrm{~h}$ \\
\hline Chine & 46,7 & 48,7 & 36,7 & 24,6 & 34,7 & 22,6 \\
\hline Toulouse & 45,0 & 46,9 & 40,1 & 30,6 & 37,9 & 28,1 \\
\hline Arsamass & 47,3 & 49,4 & 43,6 & 37,9 & 42,4 & 34,7 \\
\hline
\end{tabular}

\subsection{Effets d'un éclairement d'abord décroissant (lumière naturelle) puis constant}

Sans intervenir dès la naissance des oisons, on peut penser accélérer l'entrée en ponte en stoppant plus ou moins tôt la décroissance des jours naturels par une supplémentation en lumière artificielle. Ce type d'intervention a souvent été étudié sur des animaux d'âges multiples (cf. $\S 2$ 2.2.) mais on ne dispose que de peu de données sur l'avancement de la seule première ponte.

Des jours constants de 12 ou $14 \mathrm{~h}$ appliqués à partir de 13 semaines d'âge par Borisov (1966; tableau 1) conduisent sensiblement à la même précocité sexuelle que les mêmes éclairements donnés dès la naissance. L'utilisation, à partir de décembre, de jours de $14 \mathrm{~h}$ ou d'un éclairage permanent conduit dans l'essai de LÜHMAN (1968; fig. 3) à une avance de la ponte d'environ un mois par rapport 
au lot témoin en lumière naturelle et à une augmentation de production d'environ 25 œufs pendant la première année. On notera que la production des deux lots expérimentaux est alors obtenue en deux pontes (printemps et automne) contre une seule chez le témoin.
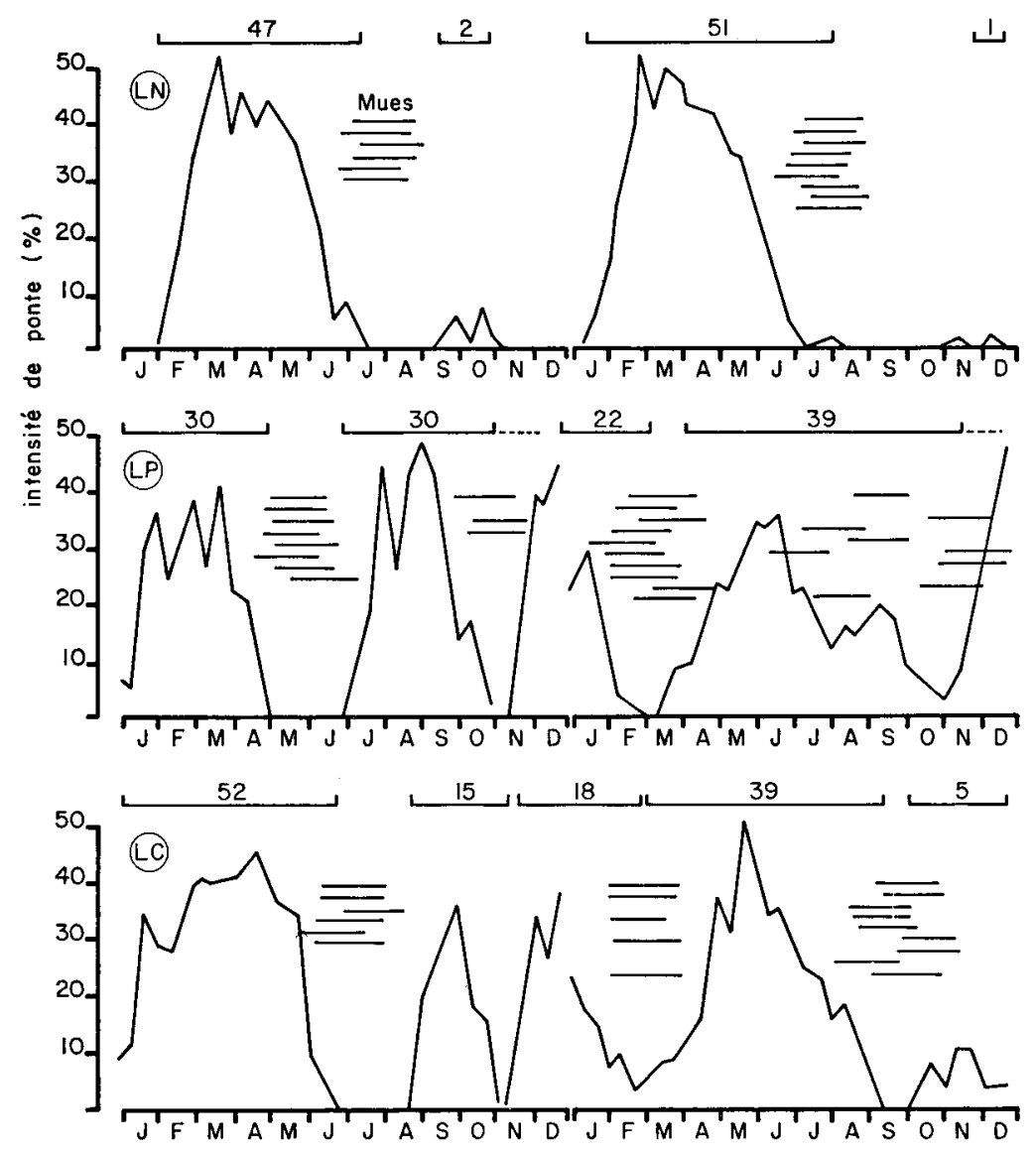

FIG. 3

Evolution de la ponte d'oies du Rhin en photopériodes constantes (d'après LuHManN, 1968).

Laying rate (p. 100) of Rhenish geese under constant photoperiods.

LN Lumière naturelle.

Natural light.

LP Lumière permanente.

Continuous lighting.

LC Lumière constante $(14 \mathrm{~h} / \mathrm{j}$ en première année; $8 \mathrm{~h} / \mathrm{j}$ en seconde année).

Constant lighting (14 h/d during the first year and $8 \mathrm{~h} / \mathrm{d}$ during the second year).

Les petits traits horizontaux représentent les périodes de mue individuelle. Le nombre total d'œufs de chaque ponte est donné au-dessus des courbes.

The small horizontal lines show the individual molting periods. The total egg production of each laying period is indicated above the curves. 
Les autres essais effectués dans le même sens (LAzAR \& SPACEK, 1978 ; RoBerTSON \& MERritT, 1979) confirment que des jours de 10 à $14 \mathrm{~h}$ appliqués en fin d'automne avancent la première ponte et en augmentent l'importance sans agir sur les performances de ponte en deuxième année.

\subsection{Effets d'un éclairement d'abord décroissant, puis croissant}

Dans les conditions de reproduction naturelle, la première ponte (fig. 1) intervient au début des jours croissants après que l'animal ait surtout subi des jours décroissants. On peut donc supposer qu'en reproduisant artificiellement une telle évolution de la durée du jour, on doit pouvoir déclencher l'entrée en ponte à un âge constant qu'elle qu'ait été la date de naissance.

Le seul essai bien développé dans ce sens est, à notre connaissance, celui de Schneider, Jurk \& Schlegel (1980) qui, à partir d'éclosions d'octobre, décembre, avril et juillet ont appliqué, en bâtiment obscur, le même programme lumineux d'abord croissant de 13 à 17 heures, décroissant ensuite de 17 à 7 heures et à nouveau croissant (fig. 4). Leurs résultats indiquent que si les 4 dates d'éclosion conduisent effectivement à une ponte satisfaisante, les animaux nés en octobre et décembre restent plus précoces que ceux des deux autres lots. La fertilité évolue aussi différemment selon les groupes, la meilleure restant celle des oies qui pondent «normalement » de février à juin. Il subsiste donc un certain effet de la saison de naissance difficile à interpréter.

\subsection{Programmes décroissants-courts puis croissants}

Afin de retarder les oisons issus d'éclosions de juillet et dont on sait que la première ponte tend à être trop précoce s'ils sont soumis aux jours naturels, ScHNEIDER, JURK \& SCHLEgel (1980) ont aussi pratiqué un maintien des animaux en jours courts avant de leur appliquer des jours croissants. Les résultats du tableau 2 montrent que cette pratique, proche de celle utilisée dans l'espèce Gallus, permet effectivement de repousser la plus grande partie de la production entre avril et fin juillet avec un supplément d'environ 10 œufs par rapport à la performance obtenue en lumière naturelle. Ce résultat présente un intérêt particulier dans la production d'oisons à rôtir dont on sait que la demande est fortement concentrée en fin d'année.

\subsection{Conclusion partielle}

La photopériode appliquée à l'oie en phase d'élevage conditionne en partie la précocité sexuelle et les performances de première ponte. Lorsque cette photopériode est maîtrisée, il paraît subsister un effet de la date de naissance sur les performances de ponte qui reste à étudier de même que l'effet éventuel de ces programmes d'élevage sur les performances de ponte des années ultérieures.

\subsection{Programmes lumineux visant chez l'adulte à étaler la ponte normale de printemps}

Sans contrôler l'environnement lumineux des animaux depuis leur naissance, on peut penser utiliser avant ou pendant les phases de ponte «naturelle» des programmes lumineux qui augmenteraient la production. 


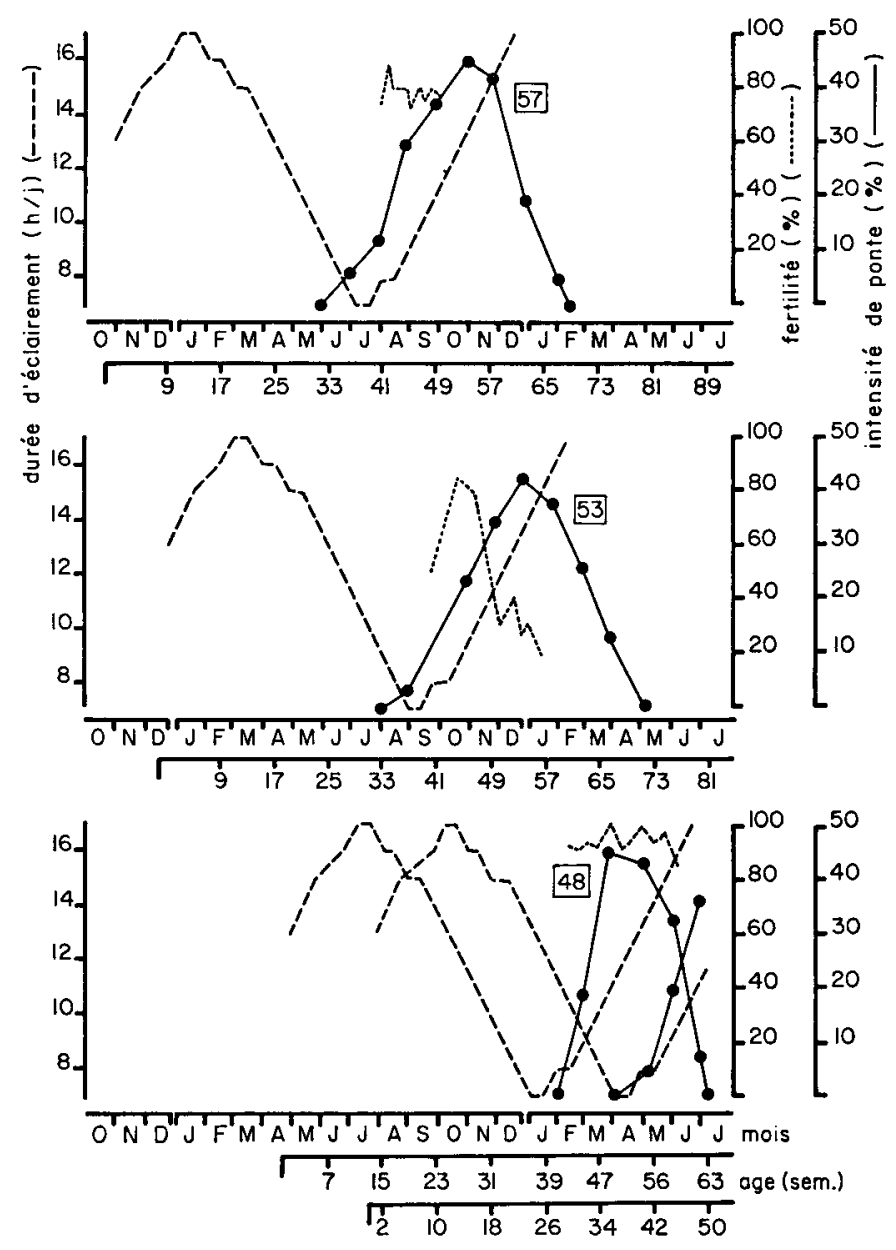

FIg. 4

Effets du mois de naissance et de programmes lumineux décroissants puis croissants sur la première ponte de l'oie

(d'après Schneider, JuRK \& SCHLEGEL, 1980; oies blanches allemandes ou italiennes).

Effects of birth month and of decreasing then increasing lighting programmes on first laying of geese (White German or Italian geese).

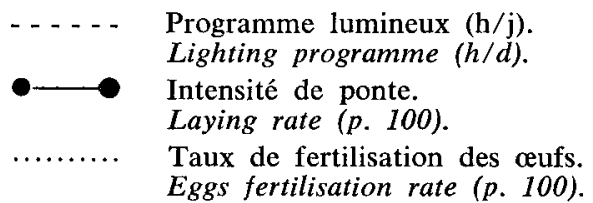

Le nombre encadré est le nombre total d'œufs produits; on ne dispose pas des résultats complets du $4^{\circ}$ lot.

The number between brackets indicates the total egg production; complete results of group 4 are not available. 
TABLEAU 2

Utilisation d'un programme lumineux décroissant - court - puis croissant pour retarder l'entrée en ponte d'oisons d'Italie nés en juillet

(SCHNEIDER, JURK \& SCHLEGEL, 1980).

Use of a decreasing lighting programme - short - then increasing in order to delay the beginning of the laying period of italian geese born in July

\begin{tabular}{|c|c|c|c|c|}
\hline \multirow[b]{2}{*}{$\begin{array}{l}\text { Période } \ldots \ldots \ldots \ldots \ldots \ldots \ldots \\
\text { Period }\end{array}$} & \multicolumn{4}{|c|}{$\begin{array}{c}\text { Nombre d'œufs/oie dans chaque période } \\
\text { Egg production/goose }\end{array}$} \\
\hline & $24 / 2-16 / 3$ & $17 / 3-10 / 4$ & $11 / 4-3 / 8$ & Total \\
\hline $\begin{array}{l}\text { Age (semaines) } \ldots \ldots \ldots \ldots \\
\text { Age (weeks) }\end{array}$ & $32-35$ & $35-39$ & $39-55$ & $32-55$ \\
\hline $\begin{array}{l}\text { Lot témoin (lumière naturelle) } \\
\text { Control group (natural light) }\end{array}$ & 5,7 & 12,4 & 25,0 & 43,1 \\
\hline $\begin{array}{l}\text { Lot retardé (1) } \ldots \ldots \ldots \ldots \\
\text { Delayed group }\end{array}$ & 2,2 & 9,0 & 43,1 & 54,3 \\
\hline
\end{tabular}

(1) Lumière naturelle décroissante de 14 à $7 \mathrm{~h}$ jusqu'à 26 semaines, $7 \mathrm{~h} / \mathrm{j}$. de 26 à 39 semaines puis jours croissants de 7 à $14 \mathrm{~h} / \mathrm{j}$. à raison de $2 \mathrm{~h} /$ mois environ.

Naturally decreasing light till 26 weeks of age; $7 \mathrm{~h} / \mathrm{d}$. of lighting between 26 and 39 weeks and then increasing days from 7 to $14 \mathrm{~h} / \mathrm{d}$. at the rate of $2 \mathrm{~h} / \mathrm{month}$.

Un premier moyen de parvenir à ce but est l'utilisation de jours courts constants $(8 \mathrm{~h})$ qui semblent permettre une prolongation de la ponte sans en affecter l'intensité (Vunder \& Krasnova, 1954 ; Schildmacher \& Rautenberg, 1957 ; Szel-Zeri, 1968). Dans l'essai récent de SChneider, Jurk \& Schlegel (1980), la prolongation est de 10 à 20 jours et s'accompagne d'une augmentation de production de 25 p. 100.

L'avancement de la ponte normale de printemps des animaux de 2 et 3 ans par utilisation de longs jours (13 à $16 \mathrm{~h}$ ) en fin d'automne est une technique mieux connue (HoffmanN \& HancocQ, 1950 ; Novikov, 1953 ; Kopylov, 1957 ; Borisov, 1958). Elle se traduit par une augmentation du nombre d'œufs produits (LüHMANN, 1968 ; BOGRE, 1980), souvent grâce à l'obtention d'une deuxième ponte en automne.

Le délai qui sépare le début de la stimulation lumineuse de la ponte du premier œuf varie de 3 à 7 semaines. Selon Szumovski (1960) il dépendrait partiellement de l'intensité lumineuse utilisée pour la stimulation mais ceci a été établi à partir de lots ne comportant que 4 animaux. Ce délai ne semble pas affecté par un maintien des oies en jours courts avant le début de la stimulation (KINNEY, Burger \& SHoFFNER, 1959).

La durée optimale de photopériode utilisable pour avancer la saison de ponte semble être de 14-15 h : des jours plus longs (17 h par exemple) favorisent l'apparition d'arrêts de ponte et de couvaison (HoffmanN \& Hancock, 1950; KopYLov, 1957). On ignore si l'usage de jours croissants ne serait pas préférable à celui de jours brusquement longs pour provoquer la stimulation initiale. 


\subsection{Obtention chez un même troupeau de deux pontes par an}

\subsection{Pontes de printemps et d'automne}

L'intervention d'une deuxième ponte annuelle a déjà été évoquée à deux reprises. Son intérêt est évidemment considérable si les æufs ainsi produits viennent réellement s'ajouter à ceux obtenus au printemps sans que la production ultérieure ne soit affectée.
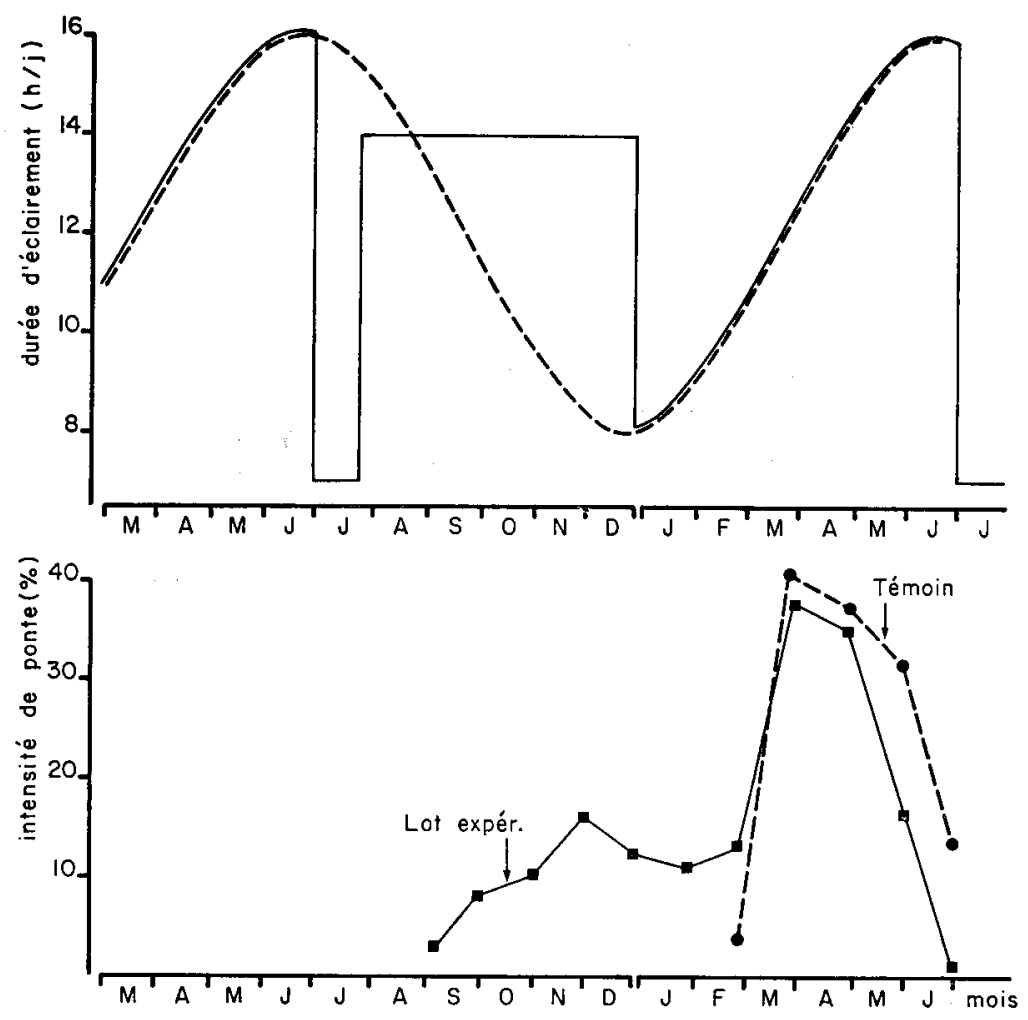

FIG. 5

Programme lumineux induisant l'apparition à partir de l'automne d'une ponte d'un an chez l'oie (d'après SAALEEV \& IONOVA, 1971; oies de Chine de 2-3 ans).

Lighting programme inducing from the autumn a one-year laying period (2-3 year old Chinese geese).

---- Durée du jour naturel $(\mathrm{h} / \mathrm{j})$.

Natural day length $(h / d)$.

- Programme lumineux $(\mathrm{h} / \mathrm{j})$.

Lighting programme $(h / d)$.

$\bullet-\longrightarrow$ Intensité de ponte, lot témoin.

Laying rate of the control group (p. 100).

- Intensité de ponte, lot éclairé.

Laying rate of the lighted group (p. 100). 
Avant d'examiner les travaux ayant spécialement porté sur le déclenchement d'une ponte à contre-saison, on peut donc rappeler que le simple avancement de la ponte de printemps par utilisation de jours de $14 \mathrm{~h}$ en fin d'automne peut conduire à l'obtention de deux pontes consécutives (Novikov, 1953 ; LüHmanN, 1968) séparées par une mue estivale (fig. 3). Les répercussions sur la ponte de l'année suivante semblent cependant difficiles à contrôler et il paraît plus sûr d'utiliser des programmes lumineux induisant une ponte d'automne après un passage d'été en jours courts.

Des oies soumises à des jours courts (de 6 à $9 \mathrm{~h}$ ) en juillet, puis stimulées par une nouvelle augmentation de la durée du jour jusqu'à $14 \mathrm{~h}$, commencent en effet une nouvelle ponte à l'automne. Celle-ci peut se limiter à la production de 15 à 30 æufs (Kociova, Ledec \& Grom, 1971 ; KaTz, 1970 ; Kosov, 1977) ou se poursuivre, dans certains cas, pendant tout l'hiver pour s'enchaîner avec la ponte du printemps suivant (Monachon \& Larrue, 1968 ; Saleev \& Ionova, 1971 - fig. 5 ; Emanovic et al., 1979). Dans tous les cas le nombre total d'œufs produits par an est significativement augmenté.

La durée des jours courts le plus souvent utilisée par les auteurs est de $7 \mathrm{~h}$; BOGRE (1973), dans un essai particulièrement digne d'attention puisque portant sur 2049 oies, obtient également de bons résultats en utilisant l'obscurité complète pendant 19 jours pour les mâles et 40 jours pour les femelles : la ponte enregistrée de septembre à janvier atteint 51 œufs/oie (contre 34 en ponte de printemps préalable) avec une fertilité de 91 p. 100.

Le rythme d'accroissement à appliquer pour passer de 7 à $14 \mathrm{~h}$ de lumière/jour paraît mal défini. Kosov (1977), Clauss \& Gehre (1978) et Bielinski (1980) utilisent respectivement 60,30 et 10 minutes/jour mais aucun de ces auteurs n'a, dans un même essai, comparé plusieurs rythmes de stimulation. Seul un résultat de Einarsson (1980) permet de penser que les progressions lentes pourraient être préférables.

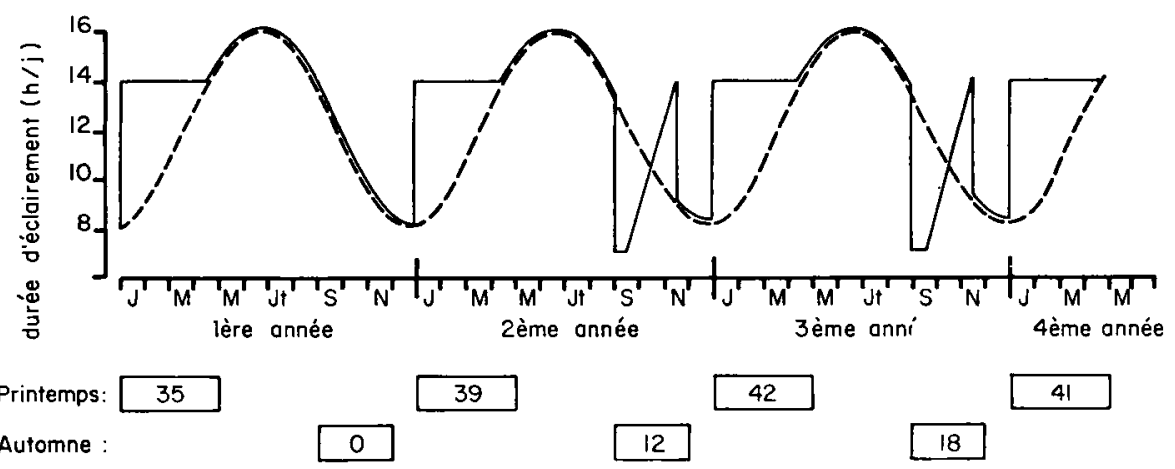

FIG. 6

Programme lumineux appliqué pour obtenir une succession régulière de pontes de printemps et d'automne chez l'oie. Effet sur le nombre d'aufs produits (en encadré)

(d'après STASKo \& MAJNA, 1976; oies d'Italie $\times$ Rhin).

Lighting programme used to obtain successive spring and autumn laying periods;

effects on egg production (numbers between brackets) (Italian $\times$ Rhenish geese).

-...- Durée du jour naturel $(\mathbf{h} / \mathrm{j})$.

Natural day length $(h / d)$.

Programme lumineux $(\mathrm{h} / \mathrm{j})$.

Lighting programme $(h / d)$. 
L'intervalle qui sépare exactement les pontes de printemps et d'automne est rarement signalé par les auteurs. Selon EINARsson (1980) \& BiELINSKI (1980) il serait de 11 à 12 semaines c'est-à-dire identique au temps de repos utilisé par ailleurs chez la cane de Barbarie, et proche de la durée de la période réfractaire des jars (revoir $\$ 1.2$.).

L'âge des oies ne paraît pas conditionner de façon claire leur aptitude à pondre en automne : STASKo \& MAJNA (1976) signalent en effet une augmentation de la production avec l'âge (fig. 6) mais ceci ne ressort pas des résultats récents de BIELINSKI et al. (1981) par ailleurs bien supérieurs à ceux des auteurs précédents.

Le taux de fertilisation des œufs d'automne est toujours au moins égal à celui des oufs de printemps (Miklosne, Ballay \& HorN, 1976 ; BielinsKI, 1980 ; EINARSSON, 1980).

\subsection{Pontes successives totalement désaisonnalisées}

Il est supposé dans le paragraphe précédent que les deux pontes annuelles se situent au printemps et à l'automne, ce qui permet de conserver la mue d'été. Si on veut réellement aboutir à une production d'oisons continue, il faut envisager qu'un troupeau au moins par atelier se reproduise en été.

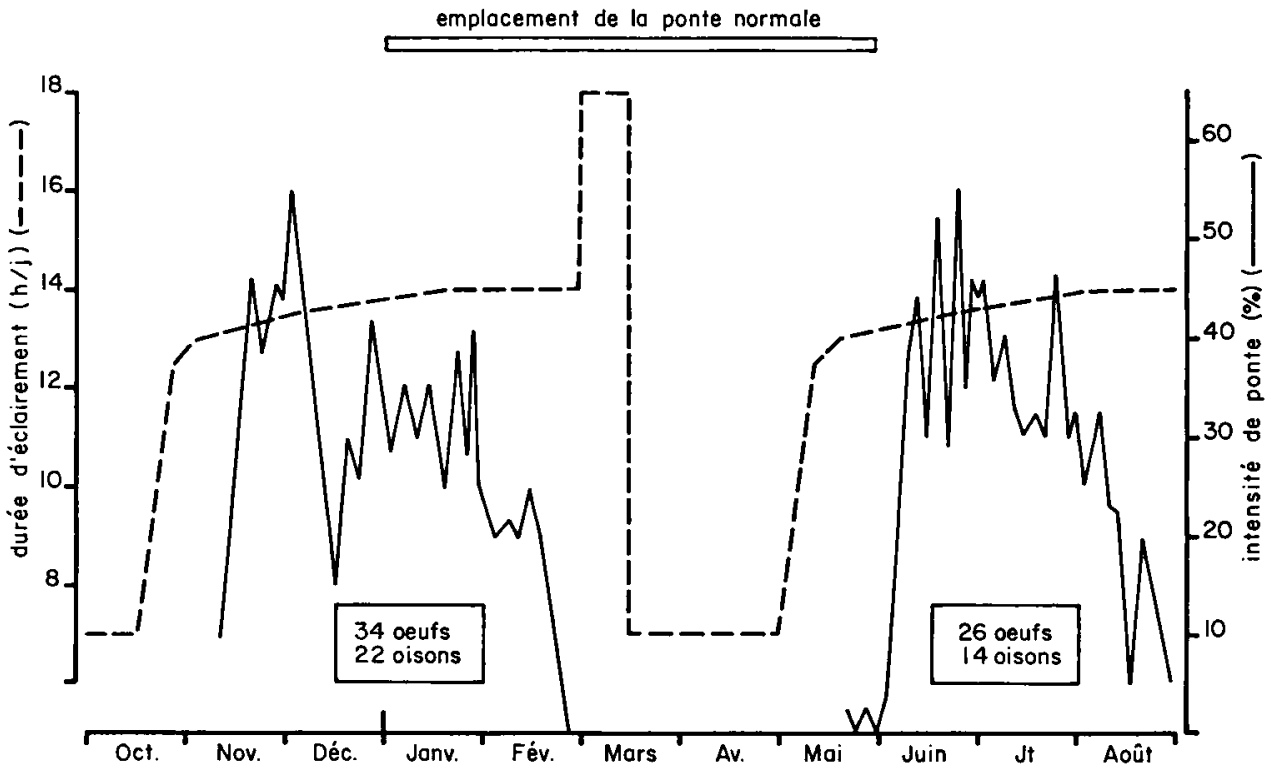

FIG. 7

Obtention d'une ponte d'été chez l'oie après repos de printemps (d'après Rousselot-PAILlEY, 1974).

Induction of a summer laying period after a short-day treatment during spring.

...... Programme lumineux $(\mathrm{h} / \mathrm{j})$.

Lighting programme $(h / d)$.

Intensité de ponte.

Laying rate (p. 100).

The open bar indicates the normal laying period. 
Ceci peut être obtenu en première ponte si l'on dispose d'oisons nés en juillet et soumis, en phase d'élevage, à un programme lumineux retardateur de la maturité sexuelle (revoir § 2.14.). En dehors de ce cas particulier, il semble, d'après Hudsky \& Lautner (1974) ou Rousselot-Pailley (1974; fig. 7) qu'une ponte d'été (juinjuillet-août) puisse être obtenue en décalant vers l'avant la ponte normale de printemps et en la faisant suivre d'un passage de 8 à 12 semaines en jours courts. RousselotPailley (1974) obtient ainsi 34 oufs et 22 oisons (65 p. 100) entre novembre et février, contre 26 et 14 (54 p. 100) entre juin et août. Ce résultat est encourageant mais il montre qu'il subsiste un problème à la fois de ponte et de fertilité lors d'une reproduction estivale.

Peut-être faut-il voir là un effet propre de la température ambiante, non évoquée jusque-là, puisque l'hypothèse a été émise selon laquelle des températures supérieures à 20 ou $25^{\circ} \mathrm{C}$ nuiraient à la reproduction des oies tant femelles (Grom, 1969) que mâles (Ruuss, 1960 ; DonTscheri-Doncev, 1969).

\section{Réponses des jars à différents programmes d'éclairement}

Les travaux portant spécifiquement sur la photosensibilité des jars n'ont été, à notre connaissance, que très peu développés. Pour la phase de croissance, on sait que, chez des jars placés en jours constants de $9 \mathrm{~h}$, la croissance testiculaire est d'abord stimulée (poids maximum à 26 semaines) mais que les testicules régressent immédiatement ; en jours constants de 14 heures, la croissance testiculaire est considérable jusqu'à 43 semaines mais on ignore le devenir de ces animaux (BlagoDATSKAIA, 1955).

Avec des jars adultes, sans préciser le «passé lumineux» des animaux, BıELINSKI (1980) montre que des jours constants de 10 ou de 12 heures assurent une meilleure persistance de production de sperme que les jours croissants naturels. Ceci confirme l'observation plus ancienne de Sergeev, Bondarenko et Kosov (1976) faite avec $14 \mathrm{~h}$ de lumière par jour.

En ce qui concerne l'effet sur les mâles des jours courts destinés à induire les arrêts de ponte des femelles, Kovacs (1971) montre que 3 semaines de jours courts $(6 \mathrm{~h} / \mathrm{j})$ entre le $1^{\text {er }}$ et le 21 novembre, suivis de jours de $14 \mathrm{~h}$ conduisent à la plus longue période de production de sperme (136 jours). De même la mise en jours courts l'été se traduirait par une bonne production spermatique après remise en jours longs d'automne.

Ceci paraît confirmé par les résultats satisfaisants de fertilité des oeufs généralement enregistrée lors des pontes d'automne (revoir \$ 2.31.) et tend à montrer que la sensibilité photopériodique des mâles ne devrait pas constituer un obstacle à l'obtention d'oisons «hors-saison».

\section{Conclusion générale}

Pour obtenir un étalement de la production d'oisons au cours de l'année, on dispose de moyens plus ou moins efficaces qui sont, en théorie au moins et pas de façon cumulative : 
- l'avancement de la ponte par des jours longs ;

- la prolongation de cette ponte par des jours courts ; croissants ;

- le décalage des entrées en ponte par des programmes décroissants, puis

- l'obtention d'une ponte d'automne par emploi de jours courts d'été suivis d'une stimulation;

- l'obtention de successions de pontes désaisonnalisées.

De nombreux points restent cependant inconnus qui ont été notés dans les conclusions partielles de chaque chapitre. On soulignera pour conclure :

- la nécessité d'études à plus long terme que celui généralement utilisé ;

- l'importance de la fertilité mâle quelquefois négligée dans les travaux de désaisonnalisation de la production;

- la totale ignorance des besoins nutritionnels liés à ces successions de phases de reproduction et de repos;

- l'utilité de mieux séparer les actions de la lumière et de la température.

Ce sont les réponses apportées à ces différentes questions qui devraient permettre à l'avenir une rationalisation progressive de la production d'oisons.

Accepté pour publication en mars 1982.

\section{Summary \\ Extension of the breeding season in geese by lighting programmes. A review}

In natural light conditions, in France the laying period of geese is located between january and june (fig. 1). In both sexes, sexual maturity is linked to day length after the age of 20 weeks (fig. 2). The spermatogenesis of the gander is also maximum between february and april whereafter the libido decreases. A molt takes place during the summer and the photorefractory period then lasts 100-110 days.

Onset of first laying can be advanced by using long days (10-14 h) during the fall (fig. 3). On the contrary, use of decreasing then increasing days separated by a short-day period delays the onset of first spring laying (fig. 4). The sexual maturity of the gander can also be advanced by using long or increasing days.

Photoperiods of $14-15 \mathrm{~h}$ are convenient to stimulate females at the end of autumn while longer days $(17-18 \mathrm{~h})$ increase subsequent broodiness. Short days $(8 \mathrm{~h})$ used during the whole breeding season lead to a small lengthening of the laying period and a better maintenance of sperm production.

Use of short days of $7 \mathrm{~h}$ and feed restriction during the summer followed by light stimulation results in a laying of 10 to 25 eggs during autumn and winter (fig. 5-6). The length of the short-day period should be about three months. In the gander, such a treatment increases sperm production and fertilization rate of out-of season eggs is generally good.

Using appropriate housing conditions, it would most likely be possible to obtain successive laying periods in alternative with short-day treatments (fig. 7). However, the accurate effects of the other climatic parameters (especially temperature) upon reproduction of geese during the summer, shoult be examined. 


\section{Références bibliographiques}

BIELINSKI K., 1980. Information about the work of Koluda Wielka experimental station in the field of goose breeding and production. In : Breeding and geese production, Zaklad Informacji Zootech, Krakow, 243-260.

Bielinski K., Elminowska G., Bielinska K., Filus F., 1981. The results of goose eggs production in two cycles per year. Rocz. Nauk Zoot., 8, 279-289.

Blagodatskaia G.I., 1955. Rôle de l'éclairement sur la croissance et le développement sexuel des oies. Tr. Inst. Zool., 12, 68-82.

BöGRE J., 1973. Egg production in two cycles in the goose. Kisérl. Közl., B (Allattenyésztés), 65, 35-41 et Anim. Breed. Abstr., 42 (1974), n $^{\circ} 2885$.

BöGRE J., 1980. Studies on increasing egg laying performance of geese. In : Bręding and geese production, Zaklad Informacji Zootech., Krakow, 16-19.

Borisov V., 1958. Cité par Szumowski, 1960.

Borisov V., 1966. Early sexual maturity and egg production of geese. Proc. 13th Wld Poultry Cong., 138-141.

Borys H., 1979. Preliminary observations on the puberty of ganders from autumn hatching. Rocz. Nauk Zoot., 6, 43-48.

Clauss F., Gehre G., 1978. Preliminary results of a second laying period for geese at the Wermsdorf division for waterfowl of the Mockrehna State Farm. Tierzucht, 32, 403-405 et Anim. Breed. Abstr., 47 (1979), n $\mathrm{n}^{\circ} 3974$.

Dontscheri-Doncev R., 1969. Facteurs conditionnant l'activité sexuelle des jars. In : «Journées de l'oie 1967 », Bull. Soc. sci. Hyg. aliment., A.F.T.A.A. et A.F.Z., 57, 102-104.

Einarsson E.J., 1980. Two egg-laying seasons per year in the geese production. Proc. 6th Europ. Poult. Conf., vol. IV, 395-402.

Emanovic D., Timet D., Majdak I., Kljucec M., Madaras F., Herak M., Kraljevic P., GradinSKi-VrbanaC B., 1979. Changes in plasma protein concentration in geese blood plasma during natural and stress-induced laying cycle. Veterinarski Arhiv., 49 (supplement), 55-57.

Grom A., 1969. Contribution aux problèmes du développement des grands élevages d'oie en Tchécoslovaquie. In : "Journées de l'oie 1967 », Bull. Soc. sci. Hyg. aliment., A.F.T.A.A. et A.F.Z., 57, 123-125.

Hoffmann E., Hancock B., 1950. The effect of length of day on egg production of geese and guineas. Poult. Sci., 29, 469-471.

Hudsky Z., Lautner V., 1974. Avoiding seasonal variation in the production of geese. Biologizace a Chemizace Výživy Zvirat, 10, 147-153 et Anim. Breed. Abstr., 43 (1975), $\mathrm{n}^{\circ} 538$.

Katz Z., 1970. Report on the effect of dark room treatment upon seasonal laying in geese. Summaries, 14th World Poult. Cong., 901-902.

Kinney T., Burger R.E., Shoffner R.N., 1959. Response of Emden Geese to light. Poult. Sci., 38, 1469-1470.

Klosowska D., Bielinska K., KLosowski B., 1979 a. Studies on the morphological and histological development of gander gonads. Rocz. Nauk Zoot., 6, 15-28.

KlosowsKa D., BielinsKa K., KLosowsKi B., 1979 b. Morphological and histological studies of ganders testis throughout the reproductive season. Rocz. Nauk Zoot., 6, 75-87 et Anim. Breed. Abstr., 40 (5 016), 1980.

Kopylov V.I., 1957. Cité par SzumowsKi, 1960.

Kociova E., Ledec M., Grom A., 1971. Inhibition of egg production in geese by progesterone. Védecké práce-Hydinárstvo, 11, 55-62 et Anim. Breed. Abstr., 1 (1973), $n^{\circ} 1325$. 
Kosov V.N., 1977. Extending the egg laying period in geese. Kubanskii Sel'skokhozyaistvennyi Institut, 153, 23-26 et Anim. Breed. Abstr., 48 (1980), n ${ }^{\circ} 4985$.

Kovács E., 1971. Effects of artificial lighting on sperm production in ganders. Baromfupar, 18, 316-325 et Anim. Breed. Abstr., 41 (1973), $\mathrm{n}^{\circ} 435$.

KropP L.B., 1976. Increasing goose production at a profit. Feedstuffs, 48, 16-17.

LAZAR V., ŠPAčEK F., 1978. The effect of day length during rearing on the subsequent performance of geese. Zivočišna Výroba, 23, 605-615 et Anim. Breed. Abstr., 47 (1979), $n^{\circ} 4514$.

LüHmanN M., 1968. Über den Einfluss zusätzlicher Beleuchtung auf die Legeleistung von Gänsen. Arch. Geflugelk., 32, 355-371.

Merritt E.S., Lemay J.A., 1963. Age and performance in geese. World Poultr. Sci. J., 19, 191-201.

Miklósné H.E., Ballay A., Horn P., 1976. Factors affecting the fertility and early embryonic mortality of the hatching egg of geese with special reference to the time of production. Proc. 5th Europ. Poult. Conf., 1250-1255.

Monachon G., Larrue P., 1968. Utilisation pratique d'un programme lumineux pour la production d'oisons d'un jour toute l'année. Proc. 3rd Europ. Poult. Conf., 90-92.

Novikov B.V., 1953. Cité par Saleev, 1975 et Szumowki, 1960.

Robertson H.A., MerritT E.S., 1979. The effect of a $10 \mathrm{~h}$ light $-10 \mathrm{~h}$ dark photoperiod on egg production by geese. Poult. Sci., 58, 1098-1099.

Rousselot-Pailley D., 1974. L'Elevage de l'oie. Son évolution grâce à la recherche. Bull. tech. Inf. Min. Agric., 292, 1-23.

Ruuss Z.R., 1960. Cité par Dontscheri-DonceV, 1969.

Saleev P., Ionova, 1971. Cité par Saleev, 1975.

Saleev P., 1975. Ways of increasing Goose Meat Production in the U.S.S.R. World Poult. Sci. J., 31, 276-287.

Schildmacher H., Rautenberg W., 1957. Legetätigkeit und Fettansatz der Hausgans unter dem Einfluss künstlich veränderter Photoperiode. Arch. Geflügelk. Kleintierk., 6, 3-15.

Schneider K.H., JuRK H., Schlegel R., 1980. The effect of light upon the egg yield in geese. In : Breeding and geese production, Zaklad Informacji Zootech., Krakow, 49-63.

Sergeev V., Bondarenko A., Kosov V., 1976. Selection and use of ganders in artificial insemination. Ptitsevodstvo, 11, 24-25 et Anim. Breed. Abstr., 45 (1977), $\mathrm{n}^{\circ} 5680$.

StašKo J., Majna R., 1976. The relationship of the spring and autumn egg production of geese. Acta Zootechnica, Nitra, 32, 105-112.

Szel-Zeri M., 1968. Cité par Schneider et al., 1980.

Szumowski P., 1960. Déclenchement de la ponte précoce chez les oies à l'aide de l'éclairage artificiel. C.R. hebd. Acad. Agric. Fr., 46, 565-574.

Vunder, Krasnova, 1954. Cité par Saleev, 1975. 